\title{
INVOLUCRAMIENTO ESTUDIANTIL EN EL PROCESO EDUCATIVO: CUESTIONARIO PARA DETERMINAR NECESIDADES DE CAPACITACIÓN DE DOCENTES
}

STUDENT ENGAGEMENT: QUESTIONNAIRE FOR THE ASSESSMENT OF TEACHERS' NEEDS IN CONTINUING EDUCATION

\author{
Volumen 13, Número 2 \\ Mayo - Agosto \\ pp. 1-23
}

Este número se publicó el 30 de mayo de 2013

Sonia Parrales Rodríguez
Jorge Robles Murillo
Irma Arguedas Negrini

Revista indizada en REDALYC, $\underline{\text { SCIELO }}$

Revista distribuida en las bases de datos:

CATÁLOGO DE LATINDEX, IRESIE, CLASE, DIALNET, DOAJ, E-REVIST@S, SHERPA/ROMEO, QUALIS, MIAR

Revista registrada en los directorios:

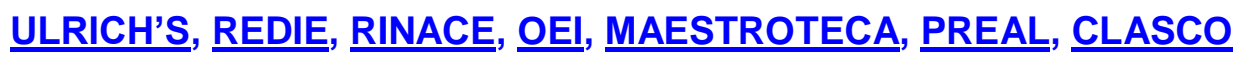




\title{
INVOLUCRAMIENTO ESTUDIANTIL EN EL PROCESO EDUCATIVO: CUESTIONARIO PARA DETERMINAR NECESIDADES DE CAPACITACIÓN DE DOCENTES

\author{
STUDENT ENGAGEMENT: QUESTIONNAIRE FOR THE ASSESSMENT OF TEACHERS' \\ NEEDS IN CONTINUING EDUCATION
}

\author{
Sonia Parrales Rodríguez ${ }^{1}$ \\ Jorge Robles Murillo \\ Irma Arguedas Negrini ${ }^{3}$
}

\begin{abstract}
Resumen: Este artículo presenta los resultados de una investigación realizada durante los años 2011-2012 con una muestra representativa de docentes de la Región Educativa de Cartago en la primera medición, y docentes de cinco colegios de San José en una segunda fase. La metodología utilizada es cuantitativa; se realizó un análisis factorial exploratorio para elaborar una estructura que mide necesidades de capacitación del personal docente de secundaria, específicamente en el área de promoción del involucramiento del estudiantado en el proceso educativo, debido a la importancia de la figura docente en este fenómeno, y su relación con el logro escolar y la permanencia en el colegio. La primera prueba fue aplicada a 365 docentes; luego de analizar las respuestas de los ítemes, se propuso un cuestionario estableciendo dos categorías. La primera es las estrategias para la promoción del logro, la cual incluye los factores condicionantes que favorecen el proceso formativo, apoyo al logro y aspectos pedagógicos, y se relacionó el $73 \%$ de las y los docentes con estas acciones. El porcentaje restante (27\%) se relacionó con acciones que enriquecen el ambiente institucional que es la segunda categoría. Producto del análisis de los resultados de esta primera etapa, se elaboró un cuestionario que discriminó los ítemes con porcentajes bajos en la estructura factorial. Esta prueba fue aplicada a 90 docentes de San José. En conclusión, se propone un cuestionario de 26 ítemes que mide los cuatro factores identificados para ser utilizado por profesionales en Orientación en el planeamiento de capacitaciones para el profesorado.
\end{abstract}

Palabras clave: EDUCACIÓN CONTINUA, PERMANENCIA EN EDUCACIÓN SECUNDARIA, PROCESO EDUCATIVO, ORIENTACIÓN EDUCATIVA, COSTA RICA

\begin{abstract}
This paper presents the results of a study that took place between 2011 and 2012. In a first phase, participants were a representative sample of secondary school teachers from the Educational Region of Cartago; the second phase took place in San José, with the participation of teachers from five secondary schools. A quantitative methodology was used; an exploratory factor analysis was run to determine a structure that measures the educators' professional development needs, in relation to the promotion of student engagement, because of the importance of educators in this phenomenon, and because of its relation to school success and completion. First, the questionnaire was applied to 365 teachers and the analysis of responses led to the construction of another version of the questionnaire based on two categories. The first category refers to strategies for the promotion of school success and includes: conditions that enhance the formative process support for success, and pedagogical aspects; $73 \%$ of participants fell into this category. The remaining percentage of participants (27\%) emphasize on actions related to the enrichment of the school climate. After the first application, a more consistent and reduced version that discriminates items with low percentages in the factorial structure was constructed. This was the version that was applied to the teachers from San José ( $n=90)$. In conclusion, a 26 item questionnaire that measures the four identified factors is proposed to be used by school counselors in their planning of consultations with secondary school teachers.
\end{abstract}

Keywords: CONTINUING EDUCATION, SCHOOL COMPLETION, SCHOOL COUNSELING, COSTA RICA

\footnotetext{
${ }^{1}$ Profesora de la Escuela de Orientación y Educación Especial, Universidad de Costa Rica. Licenciada en Educación con Énfasis en Orientación, Bachiller en Estadística. Dirección electrónica: sonia.parrales@ucr.ac.cr

2 Profesor de la Escuela de Orientación y Educación Especial, Universidad de Costa Rica y Orientador del CTP San Juan Sur. Máster en Trastornos de Aprendizaje con Énfasis Neurológico, Licenciado en Educación con Énfasis en Orientación.Dirección electrónica: jorge.robles@ucr.ac.cr.

${ }^{3}$ Profesora de la de la Escuela de Orientación y Educación Especial, Universidad de Costa Rica. Máster en Orientación Escolar, Bachiller en Enfermería. Dirección electrónica: irma.arguedas@ucr.ac.cr.
}

Artículo recibido: 11 de enero, 2013

Aprobado: 20 de mayo, 2013 


\section{Introducción}

La importancia de construir un cuestionario que cuantifique las necesidades de capacitación del personal docente, para favorecer el involucramiento de las y los estudiantes en el proceso educativo, nace con la evaluación de los recursos que poseen estos profesionales, por medio de una investigación cualitativa que antecede a la presentada en este artículo. Los detalles de esta investigación, así como las personas participantes se mantendrán como datos generales ${ }^{4}$.

Ese estudio resaltó la importancia de la figura docente en este proceso, que conduce al logro escolar y a la permanencia en el colegio. Si bien las educadoras y los educadores que participaron en el estudio cuentan con muchos recursos, no es el caso de la totalidad del profesorado. Muchas y muchos docentes requieren capacitación que active esas potencialidades, e incluso quienes tienen los recursos reconocen la necesidad de continuar su formación para responder de forma más atinada a las necesidades que se presentan en su quehacer educativo en instituciones de secundaria.

El trabajo se llevó a cabo partiendo de que la detección de necesidades de formación favorece el planeamiento basado en el establecimiento de prioridades y la emisión de medidas pertinentes, lo que respondería al enfoque descriptivo para la capacitación descrito por Esteve, citado por Mata (2004). Este se centra en favorecer las respuestas docentes al conjunto de factores que influyen en la interacción docente-estudiante, y enfatiza en que el enfoque pedagógico es más importante que los rasgos de personalidad o los estilos de enseñanza, los cuales pueden ser variados, pero igualmente eficaces para afrontar situaciones educativas. Adicionalmente, se propone visualizar el asesoramiento como un proceso de enriquecimiento mutuo entre agentes educativos, y para la satisfacción de las necesidades de las y los estudiantes. Se trata de un trabajo colaborativo para fomentar los logros del alumnado en las áreas personal-social, educativa y vocacional, incorporando criterios, tanto desde la teoría como desde la práctica.

En este artículo se reporta el proceso de construcción y validación de un instrumento para detectar estas necesidades de capacitación.

\footnotetext{
${ }^{4}$ Información adicional de la investigación cualitativa de interés para la lectora o el lector pueden ser solicitados a las o el autor.
} 


\section{Referente teórico}

El involucramiento es el nivel de participación e interés del estudiantado hacia su proceso de aprendizaje, así como el grado de conexión que tiene con la institución educativa. Tiene componentes conductuales, cognitivos y emocionales, los cuales interactúan entre sí, reflejando la integralidad propia del ser humano (Fredricks, Blumenfeld y Paris, 2004). Para referirse al constructo involucramiento, que en inglés se denomina school engagement, también se han utilizado los términos implicación escolar, compromiso y enganche (González, 2010). Al involucramiento se le está prestando creciente atención por la relación que guarda con la prevención de la salida anticipada del sistema educativo y con los rendimientos de las alumnas y los alumnos. También, se ha observado que favorece la autonomía y el sentido de pertenencia durante la adolescencia, factores asociados con el desarrollo saludable, los logros a nivel escolar y el bienestar psicológico en esta etapa del ciclo vital (Archambault, Janosz, Morizot y Pagani, 2009).

Los factores que conducen al involucramiento son similares a aquellos que conducen a la permanencia en secundaria, los cuales, a su vez, guardan relación con las condiciones para el desarrollo saludable y la preparación para enfrentar adversidades. La tarea de ofrecer las condiciones que intervienen en el involucramiento es una responsabilidad compartida. Lo mismo puede decirse del fracaso, se refiere a bajo rendimiento, salida anticipada o rezago, el cual afecta a un número significativo de estudiantes, sobre todo, a quienes se encuentran en una situación económica desfavorable, en cuyo caso se trataría más que de un fracaso escolar, de un fracaso social (Martínez-Otero, 2009; Programa Estado de la Nación en Desarrollo Humano Sostenible, 2011).

Algunos factores personales para el involucramiento son la motivación para el logro, la cual es mediada por las aspiraciones que tengan el alumno o la alumna, y las experiencias exitosas previas. Asimismo, tener metas de aprendizaje, en contraposición a solamente metas de obtener las calificaciones necesarias, favorece la persistencia y la utilización de estrategias autorreguladoras y para la superación de obstáculos (Arguedas, 2010; Lens, Matos y Vansteenkiste, 2008). A nivel familiar son importantes un clima afectivo, la comunicación, las normas razonables y el respeto, un nivel educativo de los progenitores medio o superior y la ausencia de deficiencias acumuladas producto de la desigualdad de oportunidades tanto a nivel social como pedagógicos (Fernández, 2009). Con respecto a los factores institucionales, se han confirmado lo determinantes que son el trato respetuoso y la 
promoción de la autonomía, y el riesgo que implican la exclusión y el desafecto (Van Ryzin, Gravely y Roseth, 2009).

El rol de la persona docente en la edificación de la motivación y la promoción del involucramiento es altamente relevante. Aunque los aspectos pedagógicos y la creación de ambientes de aprendizaje estimulantes son esenciales, son igualmente necesarias las intervenciones para favorecer una autonomía responsable, el esfuerzo sostenido, la tolerancia a la ambigüedad y a la frustración y la seguridad producto de experiencias de éxito (Klem y Connell, 2004; Sánchez, 2006). Asimismo, en un estudio realizado para identificar formas en las que personal docente de secundaria promueve el involucramiento del estudiantado (Arguedas, 2011), se constató la importancia del respeto hacia el o la estudiante, del conocimiento y comprensión de las características, necesidades y experiencias de vida del alumnado, de la enseñanza relevante y del seguimiento para el logro. Adicionalmente, se determinó como favorecedor del involucramiento el tipo de docente dialogante, que establece normas de trabajo y convivencia, que muestra interés por las alumnas y los alumnos y promueve la participación. Otra práctica de docentes que favorecen el involucramiento es el balance entre el aprendizaje individual y el aprendizaje socializado, el cual favorece la motivación, el sentido de logro y el funcionamiento de las capacidades intelectuales. Algunos de estos factores son mencionados por Carazo y López (2009), al referirse a la motivación y cómo esta aumenta si lo que las profesoras y los profesores presentan está vinculado con las realidades cotidianas de sus alumnos. Estos mismos autores enfatizan en la estrecha relación entre la emoción y la cognición, lo que implica que, al influenciarse mutuamente los aspectos afectivos y cognitivos para el aprendizaje, el estudiantado requiere formación socioafectiva, experiencias significativas y retos factibles de enfrentar, todo lo cual favorece el involucramiento en las tareas y el sostenimiento del esfuerzo. Puede decirse, entonces, que los desafíos que enfrenta el profesorado para cumplir con su labor formadora son grandes. En síntesis, aparte de saber enseñar contenidos y conocer cómo aprenden sus estudiantes, es preciso que cree "situaciones de enseñanza efectivas y afectivas... y tareas contextualizadas y situadas" (Villalobos, 2011, p.1).

El proporcionar condiciones para el involucramiento es una tarea ardua y compleja, por lo que el personal docente requiere de diversos apoyos para cumplirla con eficacia; la asesoría por parte de especialistas en Orientación es uno de ellos. El asesoramiento a 
docentes es uno de los componentes principales de las responsabilidades del personal especializado en Orientación en las instituciones educativas, en función de la prevención, de la atención a la diversidad y del desarrollo integral del alumnado que persiguen los diferentes actores educativos. Es una de las intervenciones indirectas que se realiza como complemento de la atención directa al estudiantado, con miras a promover las condiciones para un desarrollo integral, la potenciación de recursos para evitar problemas o reducir su incidencia y aumentar la probabilidad de que la totalidad del alumnado aprenda a partir de su diversidad (Parras, Madrigal, Redondo, Vale y Navarro, 2008; Pereira, 2007).

Tal y como se expuso, la figura docente juega un papel trascendental en el involucramiento del estudiantado y dada esta relevancia, se han hecho estudios tanto a nivel nacional como internacional sobre las formas de promoverlo. Como una forma de apoyar en el cumplimiento de esta tarea, nos abocamos a aportar una herramienta para la detección de necesidades de capacitación del personal docente con miras a la activación de potencialidades para favorecer el involucramiento. Con base en datos cualitativos de una investigación anterior, y de otros estudios consultados, se procedió a la elaboración de un cuestionario para facilitar la identificación de tales necesidades y la consecuente oferta de opciones para su satisfacción. Los ítemes se clasificaron en dos variables: clima educativo y apoyo para el logro. El clima educativo se refiere a las características del ambiente institucional, que pueden ser de tipo estructural, regulatorio y físico y se reflejan en la sensación de bienestar o malestar, las conexiones interpersonales que se establecen, la organización, las normas y las metas educativas. Los climas que más favorecen el involucramiento y el desarrollo del estudiantado, en general, se basan en las relaciones respetuosas, favorecen la responsabilidad, son inclusivos y funcionan con base en normas que facilitan la convivencia y la productividad. El apoyo al logro es un conjunto de acciones dirigidas hacia la satisfacción de necesidades de la etapa adolescente, entre las que destacan la de pertenencia, de oportunidades para enfrentar retos alcanzables y el consiguiente sentido de competencia personal. El apoyo al logro favorece la edificación de habilidades en diversos ámbitos y el desarrollo de asociaciones positivas con el aprendizaje, al plantear tareas relevantes que favorecen la comprensión. 


\section{Método}

La elaboración y validación del cuestionario, para determinar las necesidades de capacitación del profesorado de secundaria en el área de recursos docentes para favorecer el involucramiento de estudiantes en el proceso educativo, estuvo organizada por varias etapas. La secuencia se muestra en la siguiente figura:

Figura 1. Proceso metodológico de la investigación.

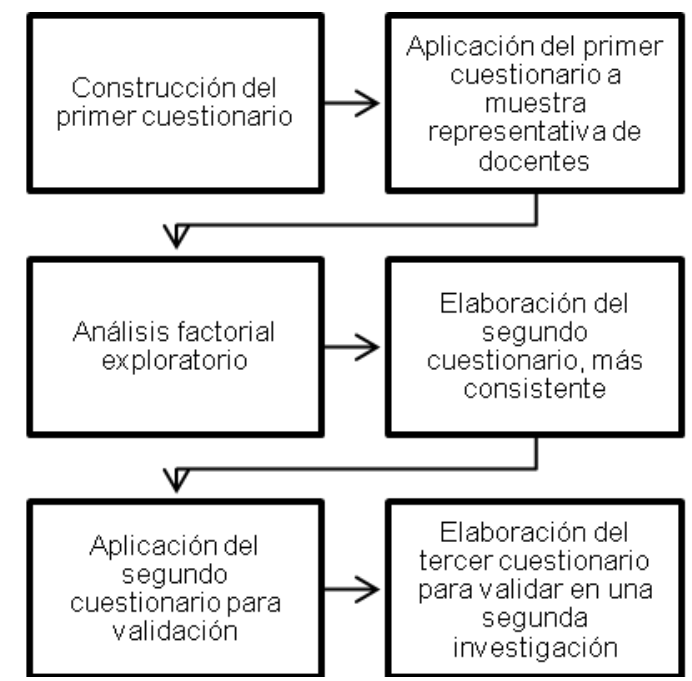

Fuente: Elaboración propia.

El primer cuestionario fue sometido a análisis factorial exploratorio y otro más consistente (producto del primero) fue aplicado a una segunda muestra para su validación.

\section{Método utilizado para la construcción y la validación del primer instrumento}

Para este proceso se siguieron los siguientes pasos:

1) Al revisar la literatura existente se determinó que un conjunto de recursos docentes ya ha sido identificado, por lo que, más que establecer evidencias de validez de contenido, la tarea en esta etapa consistió en redactar ítemes para que el profesorado indicara la medida en que según su criterio, al momento de llenar el instrumento, contaba con el recurso docente en cuestión. Se determinó el dominio de contenido con base en la literatura, porque no se trata de un constructo psicológico, sino de prácticas habituales de las y los docentes y los principios que las fundamentan. Los 
recursos docentes para favorecer el involucramiento se ubicaron en dos variables interrelacionadas: 'clima educativo' y 'apoyo al logro', coincidentes con los conocimientos disponibles a la fecha.

2) Se planteó un juicio de personas expertas para determinar, ya no la validez de contenido, sino sus opiniones en cuanto a los siguientes aspectos de cada ítem:

- Redacción (precisa y carente de ambigüedad).

- Pertinencia con el contexto de la Educación Secundaria.

- Adecuación del vocabulario a la población de interés (docentes de diversas modalidades de Educación Secundaria).

- $\quad$ Si es repetitivo.

- Si puede producir respuestas por deseabilidad social o condicionadas socialmente.

- Se refiere a una sola cosa (unidimensional).

- Utilización del lenguaje inclusivo.

- Las opciones de respuesta presentadas permiten discriminar.

- Presencia de información irrelevante.

- Si el tema tratado es factible de trabajar desde las instituciones educativas, por medio de un trabajo colaborativo entre docentes y profesionales de Orientación.

Asimismo, y debido a que el cuestionario que se construyó es para ser utilizado por profesionales en Orientación en instituciones de Educación Secundaria en la detección de necesidades de capacitación del personal docente, el grupo de personas expertas se conformó por tres profesionales en Orientación y tres docentes de Educación Secundaria, caracterizados por su interés en la formación profesional y el trabajo conjunto orientacióndocencia. Una persona de cada grupo de profesionales laboraba en una institución con horario nocturno, para de esa forma abarcar el criterio de una población más amplia. Con base en las recomendaciones aportadas por las personas expertas, se construyó la versión del instrumento que se aplicó a una muestra representativa de docentes; esta versión quedó constituida por 85 ítemes con una escala tipo Likert y dos preguntas abiertas. 
3) Este primer cuestionario se aplicó a una muestra probabilística de docentes de secundaria de la Dirección Regional de Cartago, valorada como representativa del resto del país, debido a la proporción urbano-rural y a la presencia de diversas modalidades de Educación Secundaria: académica diurna, académica nocturna, técnica diurna y técnica nocturna.

4) Para estimar los parámetros y obtener evidencias empíricas de confiabilidad y validez se utilizaron procedimientos estadísticos con ayuda del paquete SPSS. Primero se determinó el índice de confiabilidad general y de cada ítem con respecto a la totalidad. El coeficiente alfa de Cronbach resultante fue de 0,963 y mantiene la homogeneidad si se elimina el ítem. La correlación elemento total corrida es superior a 0, 276, lo que hace pensar que los reactivos miden una sola variable. Posteriormente, se hizo una reducción de datos por medio de un análisis factorial exploratorio.

En un inicio, se había establecido como una de las metas tener al menos 5 indicadores por categoría. Al tener solo dos grandes categorías, la cantidad de indicadores resultó ser mucho más grande (36 ítemes para 'clima educativo' y 98 para 'estrategias para la promoción del logro', para un total de 134 en la versión preliminar).

\section{Validación del segundo instrumento}

Una vez identificada la estructura latente originada por el análisis factorial, se rediseñó el cuestionario excluyendo los ítemes con comunalidades muy bajas y que, además, aportaban un peso muy similar en diferentes factores. Así, el cuestionario constó de 61 ítemes que miden la frecuencia con la que el personal docente realiza acciones para favorecer el involucramiento en el proceso educativo, utilizando una escala de 5 puntos.

Para la validación del segundo cuestionario se contó con la participación de docentes de cinco colegios de San José: Colegio Dr. José María Castro Madriz, C.T.P. de Educación Comercial y de Servicios, Colegio Salvador Umaña Castro, C.T.P. de Sabanilla y Colegio Medalla Milagrosa. 


\section{Participantes}

Para la selección de la muestra del primer cuestionario se utilizó el muestreo por conglomerados en una primera etapa, con una selección sistemática de las personas participantes en un segundo momento.

La Región Educativa de Cartago contaba con un total de 49 colegios, de los cuales $76 \%$ eran públicos, el $14 \%$ privado y el $10 \%$ subvencionado por el Estado. De esta forma, la subpoblación de interés se delimitó a los colegios públicos de la Región Educativa de Cartago ubicados en el Cantón Central, El Guarco, Alvarado, La Unión, Oreamuno y Paraíso, para un total de 37 instituciones educativas.

Según datos del Departamento de Estadística del Ministerio de Educación Pública (MEP), la Región Educativa de Cartago contó durante el 2010 con un total de 1336 docentes de todas las disciplinas. Para la selección de las y los docentes en cada centro educativo se utilizó el muestreo sistemático, seleccionando una unidad al azar de la lista de docentes que firmaron la lista de asistencia el día de la visita a la institución, o la lista de registro automatizado y, posteriormente, se tomaron las subsecuentes unidades hasta completar una muestra de 365 docentes.

El segundo cuestionado fue aplicado a 90 docentes de cinco colegios de San José con una selección no probabilística o dirigida.

\section{Análisis}

En primer lugar se realizó un análisis factorial para resumir y reducir los datos en una serie de dimensiones subyacentes llamadas Factores, cuya estructura agrupaba las variables que correlacionaban entre sí (Cea D’Ancona, 2004; Catena, Ramos y Trujillo, 2003). Se determinó si los resultados del cuestionario aplicado tenían la consistencia interna necesaria para ser considerado un instrumento de evaluación fiable, para lo que se utilizó el coeficiente de fiabilidad Alfa de Cronbach.

En segundo lugar se analizó la estructura con que se organizaron los contenidos del cuestionario. Para ello, se llevó a cabo un análisis factorial con el objeto de hallar las posibles combinaciones de los 85 ítemes analizados y determinar así la estructura factorial de los datos. El método utilizado fue el Análisis de Componentes Principales con rotación Varimax, que consiste en maximizar la varianza de los factores y minimizar el número de variables con saturaciones altas en un factor para una mejor interpretación. 
Después de confirmar los supuestos requeridos para un Análisis Factorial Exploratorio $\left(\mathrm{KMO}=0.904\right.$ y Test de Bartlett $\left.X^{2}(1830)=8682.044 ; \rho=0.000\right)$, se realizó el análisis excluyendo 24 ítemes con bajas puntuaciones factoriales calculadas a partir de la matriz factorial rotada y las comunalidades. Los 61 ítemes que permanecieron en la segunda versión del cuestionario se agruparon en 4 factores que explican un $41.44 \%$ de la varianza total de los puntajes obtenidos.

\section{Resultados}

Los resultados obtenidos en el primer cuestionario establecen una estructura consistente conformada por cuatro factores. A continuación, se ofrecen los coeficientes de correlación de los ítemes con el factor correspondiente, después de haber sometido las puntuaciones a una rotación varimax. En la Tabla 1 se presentan los pesos factoriales.

Tabla 1. Coeficientes que relacionan las variables observadas con los factores obtenidos.

\begin{tabular}{|ll|ll|ll|ll|}
\hline \multicolumn{2}{|c|}{ Factor 1} & \multicolumn{2}{c|}{ Factor 2} & \multicolumn{2}{c|}{ Factor 3} & \multicolumn{2}{c|}{ Factor 4} \\
ITEM78 & .767 & ITEM 21 & .636 & ITEM 82 & .718 & ITEM 31 & .614 \\
ITEM 77 & .697 & ITEM 19 & .607 & ITEM 83 & .637 & ITEM 43 & .612 \\
ITEM 79 & .696 & ITEM 15 & .602 & ITEM 81 & .606 & ITEM 44 & .585 \\
ITEM 66 & .682 & ITEM 22 & .580 & ITEM 62 & .588 & ITEM 53 & .567 \\
ITEM 68 & .665 & ITEM 20 & .577 & ITEM 60 & .576 & ITEM 34 & .538 \\
ITEM 67 & .628 & ITEM 25 & .572 & ITEM 70 & .568 & ITEM 40 & .525 \\
ITEM 76 & .614 & ITEM 14 & .569 & ITEM 16 & .506 & ITEM 51 & .477 \\
ITEM 69 & .600 & ITEM 6 & .569 & ITEM 61 & .476 & ITEM 42 & .466 \\
ITEM 64 & .513 & ITEM 26 & .537 & ITEM 41 & .472 & ITEM 63 & .448 \\
ITEM 80 & .508 & ITEM 11 & .522 & ITEM 59 & .465 & ITEM 35 & .432 \\
ITEM 48 & .504 & ITEM 27 & .494 & ITEM 3 & .464 & ITEM 36 & .404 \\
ITEM 65 & .465 & ITEM 30 & .484 & ITEM 73 & .448 & ITEM 52 & .389 \\
ITEM 54 & .456 & ITEM 13 & .454 & ITEM 56 & .416 & ITEM 75 & .380 \\
ITEM 49 & .384 & ITEM 32 & .447 & ITEM 50 & .404 & ITEM 57 & .378 \\
& & ITEM 23 & .429 & ITEM 47 & .377 & & \\
\hline
\end{tabular}

Fuente: Resultados de la encuesta realizada al grupo de docentes de la RE de Cartago (2012).

El factor uno hace referencia a las "condiciones que favorecen el proceso formativo" y contiene los siguientes ítemes: 
- 78: Conozco formas de fortalecer en el estudiantado el manejo de la frustración.

- 77: Conozco las preocupaciones que tienen mis estudiantes.

- 79: Conozco maneras de ayudarle al estudiantado a desarrollar la perseverancia.

- 66: Cuando un(a) estudiante muestra indisciplina, puedo identificar las causas.

- 68: Cuando un(a) estudiante muestra desmotivación, puedo identificar las causas.

- 67: Cuando un(a) estudiante muestra indisciplina, conozco formas de ayudarle a mejorar.

- 76: Conozco los desafíos que enfrentan mis estudiantes a nivel socioeconómico.

- 69: Cuando noto que un(a) estudiante está perdiendo el interés, sé cuáles estrategias utilizar para que lo recupere.

- 64: Promuevo la participación de las y los jóvenes en actividades recreativas.

- 80: Brindo oportunidades para aprender de las dificultades.

- 48: Mis estrategias para adaptar la enseñanza a la gran diversidad existente entre estudiantes son suficientes.

- 65: Estimulo la formación de las y los jóvenes en el área artística.

- 54: Evalúo con estrategias que favorecen la creatividad.

- 49: Identifico las fortalezas de cada estudiante.

Este factor se refiere a un conjunto de condiciones que diversos estudios (Arguedas, 2011; González, 2010) han mostrado son tan importantes para el aprendizaje y el logro como los aspectos cognitivos. Como puede verse en los 14 ítemes de este factor, se trata de aspectos socio-afectivos entre los que destacan la necesidad del manejo de la frustración, la consideración por las situaciones que aquejan al estudiantado y el fortalecimiento de la perseverancia.

Otras áreas del estudiantado que favorece el personal docente que están incluidas en este factor son el grado de interés, la motivación, la implementación de prácticas respetuosas de la diversidad y la atención de su formación integral.

El factor dos se refiere a las acciones de "apoyo al logro" y contiene los siguientes ítemes:

- 21: Si un(a) estudiante presenta una dificultad conductual, le doy seguimiento.

- 19: Si un(a) estudiante presenta una dificultad personal, le doy seguimiento. 
- 15: Superviso frecuentemente el rendimiento académico del estudiantado.

- 22: Si un (a) estudiante presenta dificultades de rendimiento, es necesario ofrecerle apoyo académico.

- 20: Si un(a) estudiante presenta una dificultad familiar, le doy seguimiento.

- 25: Resalto en el estudiantado aquellos aspectos en que han mejorado.

- 14: Tengo estrategias para aumentar la motivación de las y los estudiantes.

- 6: Implemento evaluación tanto sumativa como formativa.

- 26: Realizo actividades de aprendizaje que responden a las características del estudiantado.

- 11: Para un mayor aprendizaje identifico los conocimientos previos del estudiantado.

- 27: Realizo actividades de aprendizaje que implican participación activa del estudiantado.

- 30: Estimulo al estudiantado a ir a clases.

- 13: Tengo formas de ayudar a las y los estudiantes a mejorar en aquello que les cuesta.

- 32: Promuevo actividades que favorecen el logro académico del estudiantado.

- 23: Conozco métodos para hacer de las experiencias de aprendizaje momentos estimulantes.

- 7: Facilito que lo estudiado en clase pueda ser aplicado a la vida diaria del estudiantado.

- 10: Tomo en cuenta los intereses de las y los estudiantes a la hora de preparar la clase.

Uno de los propósitos de mayor relevancia para el personal docente en su trabajo con estudiantes es que estas y estos obtengan logros significativos en diversos ámbitos. A pesar de la relevancia, a veces se pierde de vista que para obtener logros se requieren de variados soportes (apoyo familiar, personal, para enfrentar las dificultades de rendimiento académico); en el factor dos destaca la labor del docente en la provisión de esta ayuda. Cabe agregar que lo mismo puede decirse de las y los docentes: necesitan apoyos para enfrentar la amplia gama de retos en su trabajo. 
Este factor enfatiza las acciones destinadas a darle seguimiento al estudiantado, no solamente en el ámbito académico, sino en el conductual y personal. Tener conciencia de que para que un estudiante tenga logros debe tener apoyo incluye varios elementos relacionados con la promoción de las condiciones para que los estudiantes progresen en sus estudios.

El factor tres introduce los siguientes ítemes que hacen referencia a los "aspectos pedagógicos" relacionados con la filosofía que sustenta el trabajo educativo y las metodologías utilizadas por el profesorado:

- 82: Antes de iniciar actividades, me aseguro de captar la atención de las y los estudiantes.

- 83: Al realizar actividades brindo instrucciones claras.

- 81: Aplico estrategias conocidas, variándolas según las situaciones.

- 62: Diseño actividades que comprueben la comprensión.

- 60: Fomento la aplicación de los conceptos estudiados.

- 70: Cuando defino objetivos de aprendizaje, identifico los pasos necesarios para alcanzarlos.

- 16: Cuando inicio una lección tengo preparadas las actividades de aprendizaje que van a permitir alcanzar los objetivos.

- 61: Divido las asignaciones en tractos alcanzables.

- 41: Uso ejemplos interesantes para el estudiantado.

- 59: Transmito a las y los estudiantes que el esfuerzo promueve la mejoría.

- 3: Utilizo estrategias durante las lecciones para que las y los estudiantes mantengan la concentración.

- 73: Consigo cumplir con lo planeado sin necesidad de amenazar a las y los estudiantes.

- 56: Favorezco que las y los estudiantes se sientan a gusto cuando están aprendiendo.

- 50: Asigno trabajos que el estudiantado pueda cumplir.

- 47: Las normas que se establecen en clase son para facilitar el trabajo.

- 5: Aplico actividades de aprendizaje en las que se utilizan varios sentidos (elementos auditivos, visuales y kinestésicos). 
Destacan el planeamiento y la preparación, además del manejo de aula por parte del y la docente. La persona ubicada en esta área de fortaleza hace evidente su gusto por enseñar y favorece la satisfacción por aprender. Este factor incluye lo relacionado con la innovación y el uso de elementos que facilitan el aprendizaje, tales como: partir de los conocimientos e intereses de las y los estudiantes, realizar actividades variadas. Puede incluir el uso de material atractivo y tecnologías.

El factor cuatro es el que incluye los elementos del "clima institucional", del aula en particular y de la institución, en general, que caracterizan las relaciones interpersonales en el colegio:

- 31: Promuevo condiciones para que las relaciones entre estudiantes sean cordiales.

- 43: Me preocupo por mantener buenas relaciones con mis compañeros(a) de trabajo.

- 44: Me involucro en diversas actividades de la institución.

- 43: Favorezco que cada estudiante se sienta parte importante del colegio.

- 34: Cuando hay conflictos en el aula o la institución promuevo una resolución pacífica.

- 40: Recurro al diálogo para resolver desacuerdos.

- 51: Favorezco que las y los estudiantes pierdan el miedo a equivocarse.

- 42: Muestro interés por la totalidad del estudiantado, no solo por quienes están a mi cargo.

- 63: Favorezco la participación de las y los jóvenes en asuntos estudiantiles dentro del colegio.

- 35: Modelo un comportamiento respetuoso frente al estudiantado.

- 36: Modelo un comportamiento auto controlado frente al estudiantado.

- 52: Favorezco que las y los estudiantes realicen proyectos de su propia iniciativa.

- 75: Me muestro como un ejemplo positivo ante las y los compañeros de trabajo.

- 57: Encuentro el equilibrio entre la exigencia y la flexibilidad.

Se resalta el fomento de la resolución pacífica de conflictos y de las relaciones respetuosas y cordiales entre docentes, entre estudiantes y docente- estudiante. Aquí cobran importancia los balances entre la exigencia y la flexibilidad y entre lo individual y lo colectivo. La persona que se ubica en esta área de fortaleza no solo se visualiza formando 
parte de las actividades institucionales, sino que incentiva al estudiantado a la participación significativa.

El Gráfico 1 muestra la asociación del grupo de docentes que participaron en la investigación con los factores identificados en el proceso anterior. Este gráfico muestra, de manera esquemática, el peso de los factores identificados, tal y como lo mostró el fundamento teórico al inicio del proyecto, se vislumbraron dos grandes grupos o variables, las relacionadas con aspectos pedagógicos y las que tienen que ver con el ambiente institucional y de aula. Posterior a la validación del instrumento, los aspectos pedagógicos quedaron ubicados en tres subgrupos: el que se denominó Apoyo al logro en el que estuvo ubicado el $0.8 \%$ de los participantes; el llamado Condiciones que favorecen el proceso formativo, con un $43.8 \%$ de la muestra y Aspectos pedagógicos con $28.5 \%$ de docentes participantes. Por ser factores que inciden directamente en el estímulo hacia un éxito escolar integral, estos tres subgrupos en conjunto pasaron a ser denominados "Motivación al logro" y en él se ubica el $73.2 \%$ de las y los docentes. En la variable ambiente se ubicó el $26.8 \%$ de participantes.

Gráfico 1. Distribución porcentual de la muestra agrupados según los factores identificados en la estructura factorial.

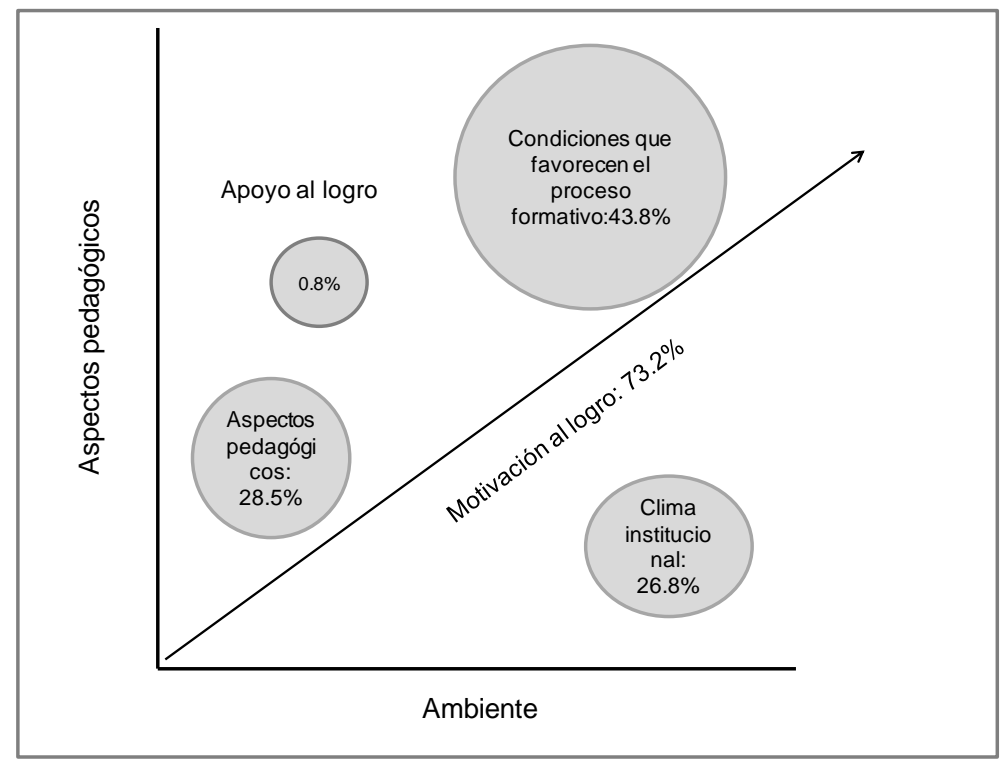

Fuente: Resultados de la encuesta realizada al grupo de docentes de la RE de Cartago (2012). 
No se encontraron diferencias estadísticas significativas al analizar estos conjuntos por las variables que identificaron al grupo de docentes participantes (sexo, edad, años de servicio docente y título más alto obtenido).

Para analizar los resultados obtenidos en la segunda aplicación del cuestionario con los 61 ítemes, se sumaron los porcentajes de respuesta del grupo de docentes que señalaron que nunca, casi nunca o a veces realiza las acciones para favorecer el involucramiento del estudiantado (Gráficos 2, 3, 4 y 5).

Gráfico 2. Porcentajes de participantes que nunca, casi nunca o a veces realiza acciones docentes para favorecer las condiciones del proceso educativo (factor 1$)$. $(n=90)$

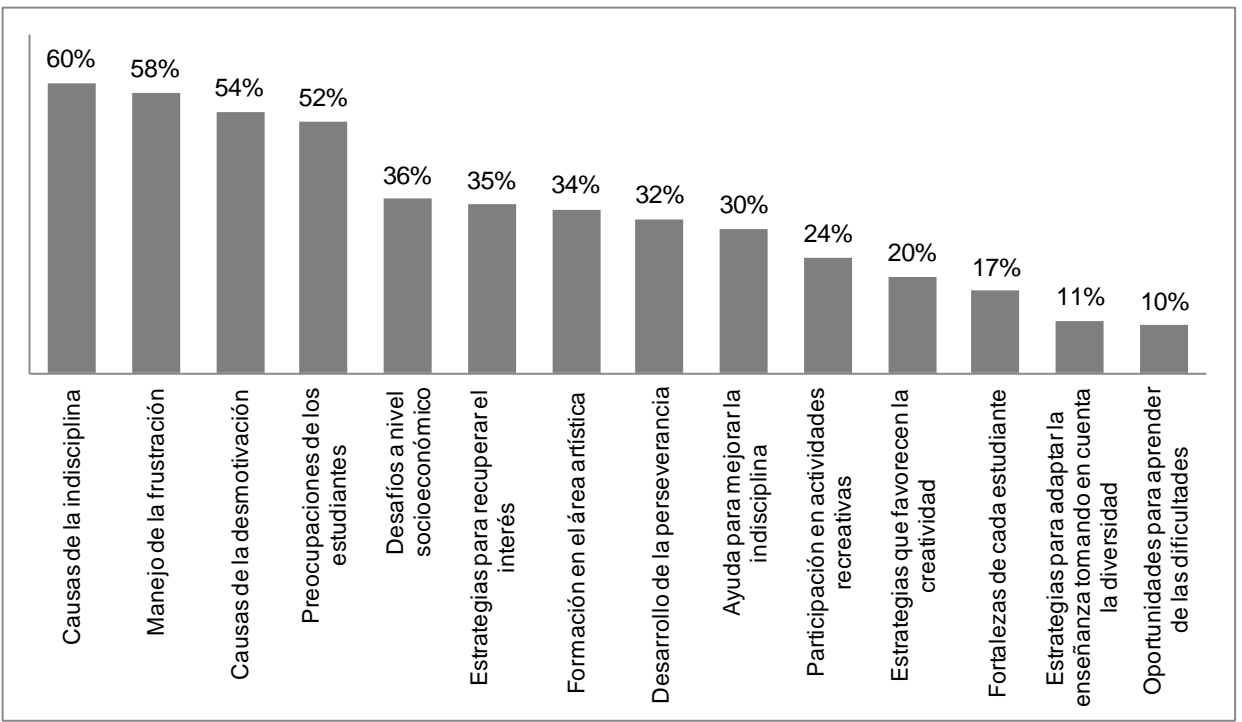

Fuente: Resultados de la encuesta realizada al grupo de docentes de San José (2012). 
Gráfico 3. Porcentajes de participantes que nunca, casi nunca o a veces realiza acciones docentes de apoyo al logro (factor 2$)$. $(n=90)$

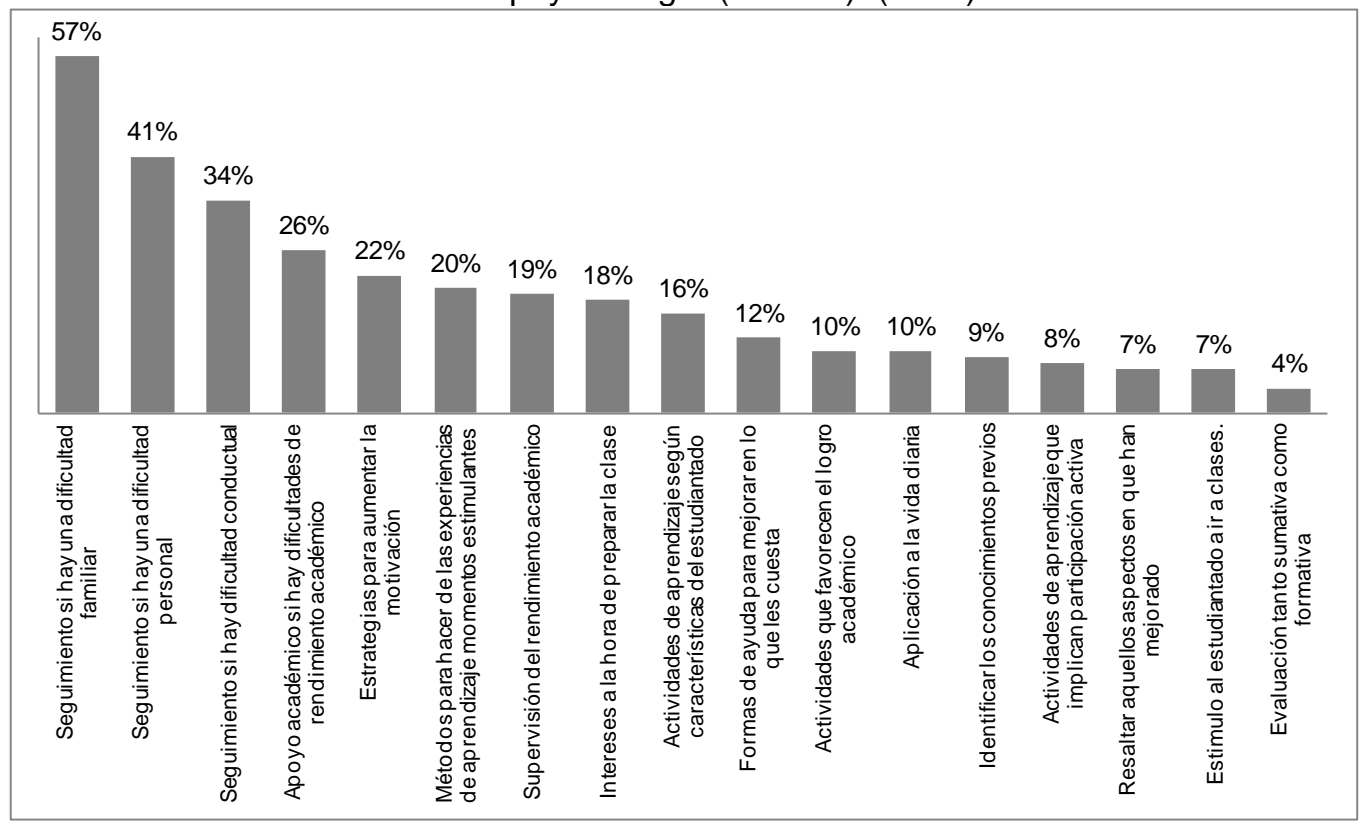

Fuente: Resultados de la encuesta realizada al grupo de docentes de San José (2012).

Gráfico 4. Porcentajes de participantes que nunca, casi nunca o a veces realiza acciones docentes que favorecen los aspectos pedagógicos (factor 3$)$. $(n=90)$

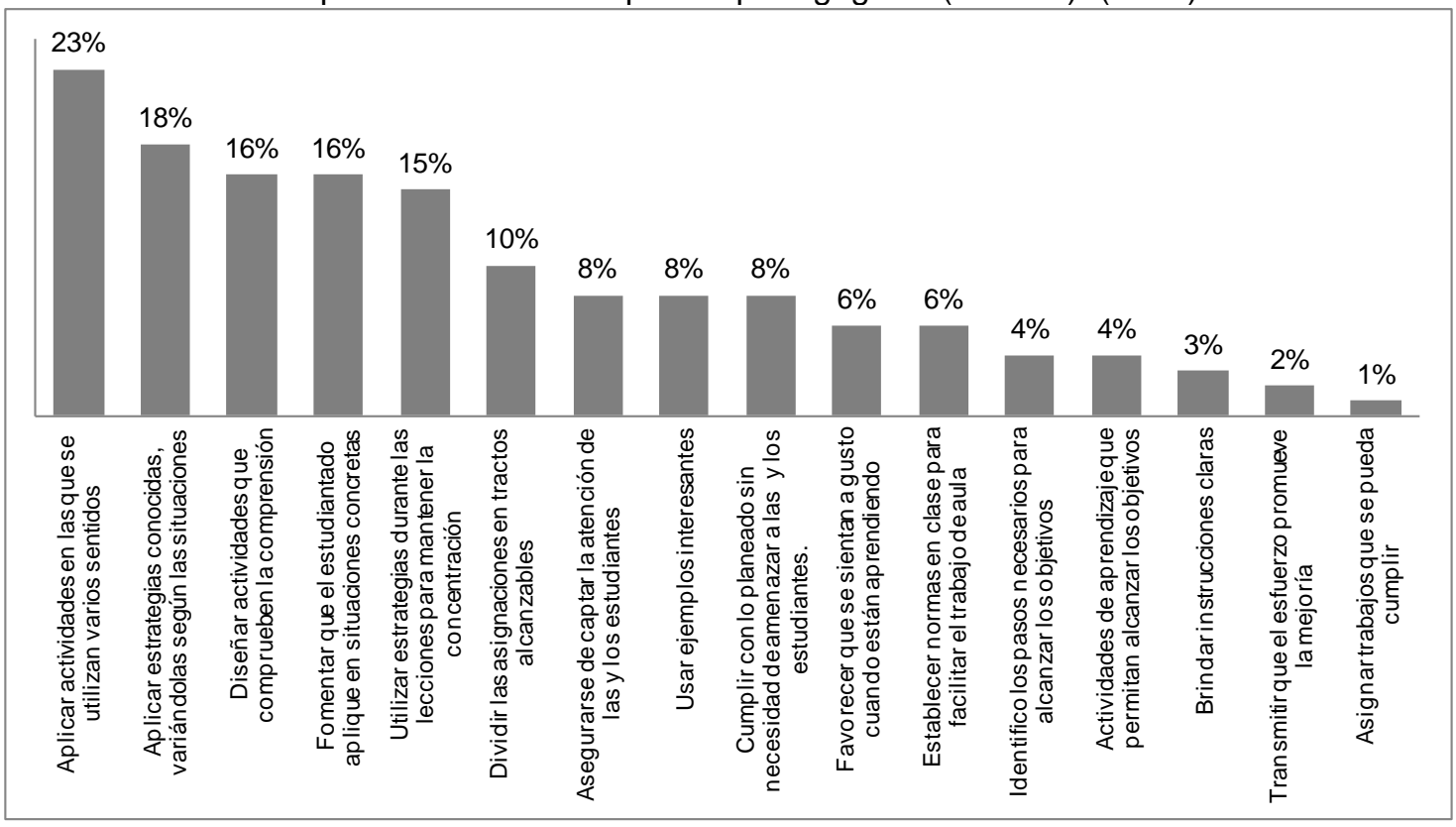

Fuente: Resultados de la encuesta realizada al grupo de docentes de San José (2012). 
Gráfico 5 Porcentajes de participantes que nunca, casi nunca o a veces realiza acciones docentes que favorecen el clima institucional (factor 4$)$. $(n=90)$

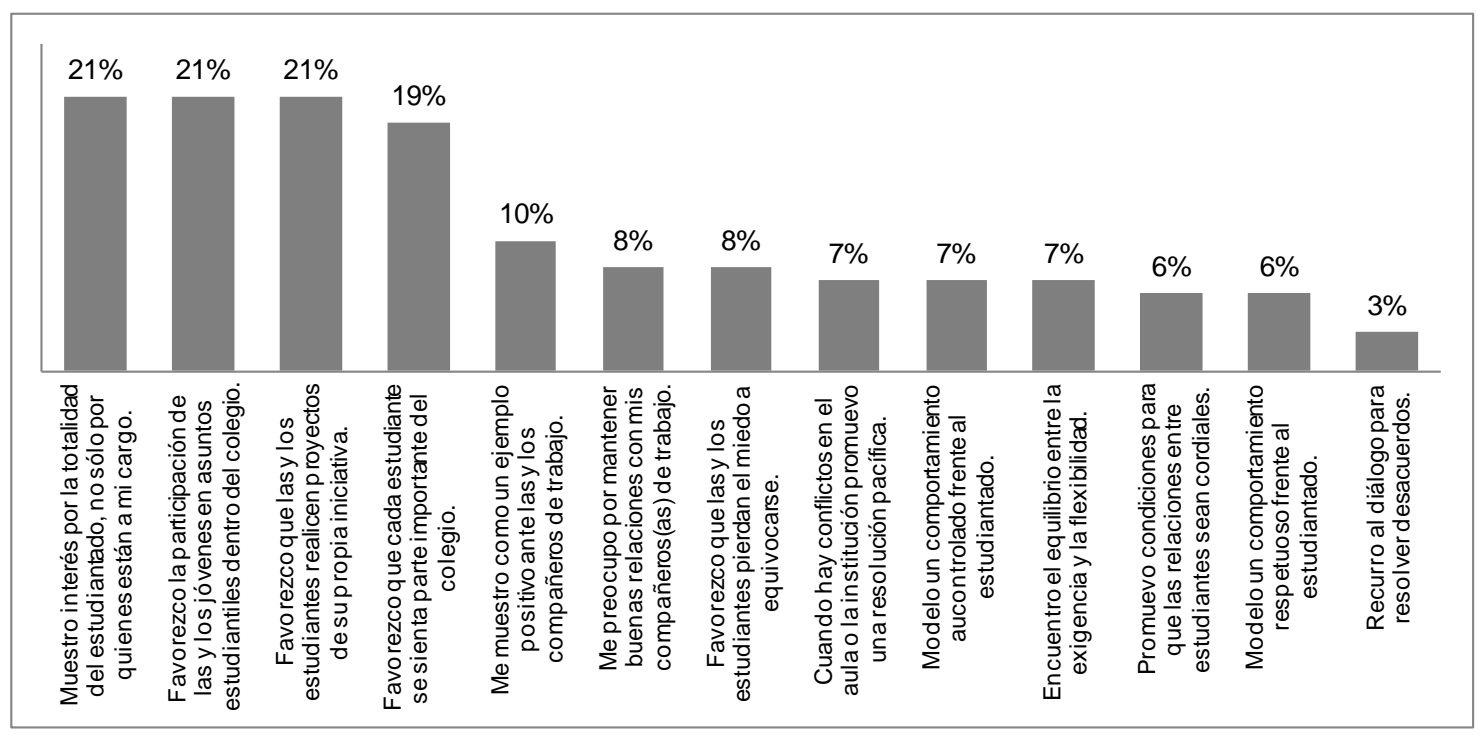

Fuente: Resultados de la encuesta realizada al grupo de docentes de San José (2012).

Fiabilidad:

Los resultados obtenidos con la muestra de 90 docentes y 61 ítemes para la fiabilidad, evaluada mediante el coeficiente alfa de Cronbach, son los siguientes:

\begin{tabular}{ll}
\hline Cuestionario total & $\alpha=0,942$ \\
Factor 1: condiciones que favorecen el & $\alpha=0,839$ \\
proceso educativo & $\alpha=0,865$ \\
Factor 2: apoyo para el logro & $\alpha=0,821$ \\
Factor 3: aspectos pedagógicos & $\alpha=0,752$ \\
\hline
\end{tabular}

Fuente: Resultados de la encuesta realizada al grupo de docentes de San José (2012).

Tal y como se desprende de los resultados anteriores, los ítemes correlacionan de manera positiva y alta. Si se elimina cualquiera de los ítemes el alfa del instrumento mantiene su homogeneidad. 


\section{Conclusiones}

El cuestionario de necesidades de capacitación del profesorado de secundaria en el área de recursos docentes para favorecer el involucramiento estudiantil en el proceso educativo identifica cuatro áreas de recursos docentes: condiciones que favorecen el proceso formativo, apoyo al logro, aspectos pedagógicos y el clima institucional.

El instrumento resumido (26 ítemes) mostró tener validez de contenido, pues el proceso de construcción fue consistente con los resultados de investigaciones anteriores y el juicio de personas expertas en el tema. Además, presentó una confiabilidad positiva y alta para el total de ítemes y para cada una de las subescalas. Esto implica que mide las dos variables en estudio (clima institucional y apoyo al logro) e identifica las necesidades en las áreas de formación docente.

Se detallan, a continuación, las 26 frases que contiene el cuestionario:

\section{Factor 1: condiciones que favorecen el proceso educativo}

1. Conozco formas de fortalecer en el estudiantado el manejo de la frustración.

2. Conozco las preocupaciones que tienen mis estudiantes.

3. Conozco maneras de ayudarle al estudiantado a desarrollar la perseverancia.

4. Cuando un(a) estudiante muestra indisciplina, puedo identificar las causas.

5. Cuando un(a) estudiante muestra desmotivación, puedo identificar las causas.

6. Cuando un(a) estudiante muestra indisciplina, conozco formas de ayudarle a mejorar.

7. Conozco los desafíos que enfrentan mis estudiantes a nivel socioeconómico.

8. Cuando noto que un(a) estudiante está perdiendo el interés, sé cuáles estrategias utilizar para que lo recupere.

9. Promuevo la participación de las y los jóvenes en actividades recreativas.

10. Estimulo la formación de las y los jóvenes en el área artística.

11. Evalúo con estrategias que favorecen la creatividad.

\section{Factor 2: apoyo al logro}

1. Si un(a) estudiante presenta una dificultad conductual, le doy seguimiento.

2. Si un(a) estudiante presenta una dificultad personal, le doy seguimiento. 
3. Si un (a) estudiante presenta dificultades de rendimiento, le ofrezco apoyo académico.

4. Si un(a) estudiante presenta una dificultad familiar, le doy seguimiento.

5. Tengo estrategias para aumentar la motivación de las y los estudiantes.

6. Realizo actividades de aprendizaje que responden a las características del estudiantado.

7. Utilizo métodos para hacer de las experiencias de aprendizaje momentos estimulantes.

\section{Factor 3: aspectos pedagógicos: 4 ítemes}

1. Aplico estrategias conocidas, variándolas según las situaciones.

2. Diseño actividades que comprueben la comprensión de la materia.

3. Cuando se estudian conceptos fomento que el estudiantado los aplique en situaciones concretas.

\section{Factor 4: clima institucional: 4 ítemes}

1. Me involucro en diversas actividades de la institución.

2. Muestro interés por la totalidad del estudiantado, no solo por quienes están a mi cargo.

3. Favorezco la participación de las y los jóvenes en asuntos estudiantiles dentro del colegio.

4. Favorezco que las y los estudiantes realicen proyectos de su propia iniciativa.

Los aspectos socio-afectivos, como conocer las formas para fortalecer en el estudiantado el manejo de la frustración, la consideración por las situaciones que aquejan al estudiantado, el fortalecimiento de la perseverancia, la comprensión de la desmotivación y de la falta de interés y razones de indisciplina en el aula, son aspectos que tienen porcentajes más bajos de frecuencia en las acciones que realiza el personal docente. De manera consistente, el $50 \%$ de las menciones de las necesidades de capacitación hace referencia a estos aspectos: "cómo trabajar con la frustración", "cómo motivar a los estudiantes", "manejo de la ira y el estrés", "técnicas de estimulación de la motivación o cambio de actitud en el aula". 
Por su lado, aspectos que tienen que ver con la atención a la formación integral como favorecer la creatividad, fomentar la participación en actividades recreativas y estimular el área artística del estudiantado son acciones que podrían requerir fortalecimiento en el grupo de docentes participantes.

El apoyo de las y los docentes ante las dificultades del alumnado motiva y ofrece oportunidades en el mejoramiento del rendimiento académico.

El tema del seguimiento a quienes muestran dificultades conductuales, personales y familiares, brindar apoyo si hay problemas de rendimiento académico e incluir actividades de aprendizaje según las características del estudiantado, de manera tal que se conviertan en momentos estimulantes y motivantes, son retos a los que se enfrenta el grupo docente participante y para lo cual requiere de más capacitación. Efectivamente, el $21 \%$ de las menciones en las necesidades de capacitación hacen referencia a este aspecto: "métodos y técnicas para un aprendizaje atractivo", "inteligencias múltiples, estilos de aprendizaje", "relaciones interpersonales en el núcleo familiar", "identificación de problemas psicosociales que puedan afectar la actitud ante la materia por parte del estudiante".

Se hace evidente que en la filosofía que sustenta el trabajo educativo, el grupo de docentes tiene gusto por la enseñanza. No obstante, incluir en el planeamiento actividades donde se usan varios sentidos, variar las estrategias según las situaciones y diseñar actividades para comprobar la comprensión de lo aprendido resultan ser prácticas que requieren ser fortalecidas por un grupo pequeño de participantes. Esta necesidad también se hace evidente en respuestas abiertas, pues algunos docentes señalan: "didáctica específica y metodología...", "estrategias de enseñanza - aprendizaje y actividades orientadas al alcance de resultados", "uso de recursos didácticos" como temas que necesitan abordar en su formación profesional.

Incentivar y favorecer a la totalidad del estudiantado en las actividades de la institución, realizando proyectos por iniciativa propia, haciéndolos sentirse parte importante del colegio, favoreciendo de esta manera el clima institucional y la relación docentes-estudiantes, así como promover el sentido de pertenencia son temas que requieren ser fortalecidos en el grupo de docentes encuestados.

Se concluye que se trata de un instrumento que posibilita la identificación tanto de fortalezas como de carencias del profesorado de secundaria, en el área de los recursos docentes para la promoción del involucramiento del estudiantado en el proceso educativo. Lo 
anterior debido a que contiene los cuatro factores identificados en un primer momento, pero con una cantidad más reducida de reactivos, que fueron seleccionados tomando como parámetro un porcentaje igual o superior a $20 \%$ en la suma de las respuestas del grupo de docentes que señalaron que nunca, casi nunca o a veces realiza las acciones para favorecer el involucramiento. Es decir, resulta tanto eficaz como eficiente, porque se logra la identificación de fortalezas y necesidades mediante la utilización de un instrumento, cuya aplicación requiere un mínimo de tiempo y contiene los ítemes que identifiquen más claramente los recursos docentes que necesitan ser atendidos.

\section{Referencias}

Archambault, Isabelle; Janosz, Michel; Morizot, Julien y Pagani, Linda. (2009). Adolescent Behavioral, Affective, and Cognitive Engagement in School: Relationship de Dropout. Journal of School Health, 79(9), 408-415.

Arguedas, Irma. (2011). Recursos docentes para favorecer el involucramiento de estudiantes de Educación Secundaria en el proceso educativo. Revista lberoamericana de Educación, 56(2).

Arguedas, Irma. (2010). Promoción de la Permanencia de Estudiantes en la Educación Secundaria. Manual de Temas y Estrategias. Instituto de Investigación en Educación. San José, Costa Rica: Editorial de la Universidad de Costa Rica.

Carazo, Viviana y López, Luis F. (2009). Aprendizaje, Coevolución Neuroambiental (Colección Pedagógica Formación Inicial de Docentes Centroamericanos de Educación Básica, № 43). San José, Costa Rica: Coordinación Educativa y Cultural Centroamericana, CECC/SICA.

Catena, Andrés; Ramos, Manuel y Trujillo, Humberto. (2003). Análisis Multivariado. Un manual para investigadores. Biblioteca Nueva. Madrid, España.

Cea, D’Ancona, María Ángeles. (2004). Análisis multivariable. Teoría y práctica en la investigación social. Editorial Síntesis. Madrid.

Fernández, Tabaré. (2009). La desafiliación en la educación media en Uruguay. Revista Iberoamericana sobre Calidad, Eficacia y Cambio en Educación, 8(4), 164-179.

Fredricks, Jennifer; Blumenfeld, Phyllis; Paris, Alison. (2004). School Engagement: Potential of the concept, State of the Evidence. Review of Educational Research 74(1), 59-109.

González, Mํㅡㄹ Teresa. (2010). El alumno ante la escuela y su propio aprendizaje: Algunas líneas de investigación en torno al concepto de implicación. Revista Iberoamericana sobre Calidad, Eficacia y Cambio en Educación, 8(4), 9-31. 
Klem, Adena y Connell, James. (2004). Relationships Matter; Linking Teacher Support to Student Engagement and Achievement. Journal of School Health, 74(7), 262-273.

Lens, Willy; Matos, Lennia y Vansteenkiste, Maarten. (2008). El profesor como fuente de motivación de los estudiantes: Hablando del qué y del porqué del aprendizaje de los estudiantes. Revista Digital de Investigación en Docencia Universitaria (RIDU), 4(1). Recuperado de: http://beta.upc.edu.pe/calidadeducativa/ridu/2008/ridu5 Im.pdf

Martínez-Otero, Valentín. (2009). Diversos condicionantes del fracaso escolar en la Educación Secundaria. Revista Iberoamericana de Educación, (51), 67-85.

Mata, Alejandrina. (2004). El sentido social de la idea de un buen maestro. Revista Electrónica Actualidades Investigativas en Educación, 4(2), 1-39. Recuperado de: http://revista.inie.ucr.ac.cr/ediciones/controlador/Article/accion/show/articulo/el-sentidosocial-de-la-idea-de-un-buen-maestro.html

Parras, Antonia; Madrigal, Ana Mª; Redondo, Sara; Vale, Patricia y Navarro, Enrique. (2008). Orientación educativa: Fundamentos teóricos, modelos institucionales y nuevas perspectivas. Centro de Investigación y Documentación Educativa (CIDE) del Ministerio de Educación, Política Social y Deporte de España.

Pereira, María Teresa. (2007). Orientación educativa (9aㅗ reimpresión de la $1^{\text {a }}$ edición). San José: Editorial de la Universidad Estatal a Distancia.

Programa Estado de la Nación en Desarrollo Humano Sostenible. (2011). III Informe Estado de la Educación. San José, Costa Rica: Consejo Nacional de Rectores.

Sánchez, Miriam. (2006). De la motivación y del síndrome del fracaso. En: Sánchez, M. y López, M. (Comp.), De la motivación y del síndrome del fracaso (2ª ed., pp. 9-12). México: Universidad Autónoma de la Ciudad de México. UACM.

Van Ryzin, Mark; Gravely, Amy; Roseth, Cary (2009). Autonomy, Belongingness, and Engagement in School as Contributors to Adolescent Psychological Well-Being. Journal of Youth and Adolescence, 38(1), 1-12.

Villalobos, Ximena. (2011). Reflexión en torno a la gestión de aula y a la mejora en los procesos de enseñanza y aprendizajes. Revista Iberoamericana de Educación, 5(3), 1-7. 